\title{
Steady Flow Around a Moving Circular Pipe in a Thermo-viscous Fluid in the Absence of Pressure Gradient
}

\author{
N. Pothanna ${ }^{1 *}$, P. Aparna ${ }^{2}$ and J. Srinivas ${ }^{3}$ \\ ${ }^{1,2}$ Department of Mathematics, VNR Vignana Jyothi Institute of Engineering and \\ Technology, Hyderabad, 500090, Telangana State, India \\ pothareddy81@gmail.com
}

\begin{abstract}
The steady flow around a moving circular pipe in a thermo-viscous fluid in the absence of pressure gradient is examined in this paper. The flow under the constant temperature gradient is considered. The solutions for the velocity and temperature fields have been obtained in terms of Modified Bessel functions with appropriate boundary conditions. The Nussult number and the Drag force on the boundary have been calculated. The effect of thermo-viscous material parameters on the Drag, velocity and temperature fields have been shown in the form of graphs and discussed in the conclusions part.
\end{abstract}

Keywords: Thermal conductivity coefficient, thermo-stress viscosity coefficient, Drag

\section{Nomenclature}

$\alpha_{1}=-p:$ The Fluid Pressure

$\alpha_{3}=2 \mu: \mu$ is the coefficient of classical(Newtonian) viscosity

$\alpha_{5}=2 \mu_{c}: \mu_{c}$ is the coefficient of (Reiner-Rivlin) cross-viscosity

$\alpha_{6} \quad:$ Thermo mechanical stress interaction coefficient

$\alpha_{8} \quad:$ Thermo stress viscosity coefficient

$-\beta_{3} \quad:$ Strain thermal conductivity coefficient

$-\beta_{1}=k \quad:$ Fourier Thermal conductivity coefficient

$a_{6} \quad:$ Thermo mechanical stress interaction coefficient (non dimensional form)

$b_{3} \quad:$ Strain thermal conductivity coefficient (non dimensional form)

\section{Introduction}

The non-Newtonian nature of materials has been the subject of extensive study for over one and half centuries. It is only in last seven or eight decades that serious attempts have been made to extend these investigation in the realm of non-linearity. The failure of the linear theories in predicting to a reasonable extent the mechanical behaviour of materials such as liquid polymers, fluid plastic, the molten metals etc., subjected to stresses has 
been the motivating force behind study of the non-linear theories for material description. A non- linear generalization of the Voigt type materials was proposed by Koh Eringen[5]. Some of the non-liner theories proposed so far (listed in references) have not taken into account the strong dependence of visco-elastic behaviour upon thermal conduction i.e. interaction/interrelation between mechanical and non mechanical (such as thermal, chemical, electromagnetic etc.,) effects even though the large amount data of experimental evidence indicate a strong dependence of visco-elastic nature of the fluid upon thermal behaviour. For example materials such as solid propellants exhibit a mechanical behaviour at moderate temperature variations, where as little or no correlation between them would be observed with that under the isothermal condition.

The concept of thermo-viscous fluids which reflect the interaction between thermal and mechanical responses in fluids in motion due to external influences was introduced by Koh and Eringin[5]. Kelly[4] studied some simple shear flows of non-Newtonian second order thermo-viscous fluids . Green and Naghdi[2,3] has given a new theory on thermoviscous fluids. Nageswara Rao and Pattabhi Ramacharyulu[8] later studied some steady state problems of certain flows dealing with thermo-viscous fluids. Some more problems of thermo-viscous flows studied by Anuradha[1] and Nagaratnam[7] in different geometries. Muthuraj and Srinivas[6] studied flow of a thermo-viscous fluid through an annular tube with constriction. Nageswara rao and Pattabhi Ramacharyulu[9] examined steady flow of a second order thermo-viscous fluid over an infinite plate. Nageswara rao and Pattabhi Ramacharyulu[10] presented steady flow of a thermo-viscous fluid through straight tubes. Nageswara rao and Pattabhi Ramacharyulu[11] studied A note on steady slow motion of thermo-viscous fluid through a circular tube. Pothanna and Srinivas et al., [14] examined the problem Linearization of thermo-viscous fluid in a porous slab bounded between two fixed permeable horizontal parallel plates in the absence of thermomechanical interaction coefficient in 2014. Pothanna et al., [13,14,15] examined some steady and unsteady state problems dealing with certain flows of thermo-viscous fluids between parallel plates with various assumptions. As proposed by Koh and Eringin the stress-tensor ' $t$ ' and heat flux bi vector ' $h$ ' are expressed as polynomial functions, viz., the rate of deformation tensor ' $d$ ':

$t=\alpha_{1} I+\alpha_{3} d+\alpha_{5} d^{2}+\alpha_{6} b^{2}+\alpha_{8}(d b-b d)$

and

$h=\beta_{1} b+\beta_{3}(b d+d b)$

with

$d_{i j}=\left(u_{i, j}+u_{j, i}\right) / 2$

and thermal by gradient bi vector ' $b$ '

$b_{i j}=\epsilon_{i j k} \theta_{k}$

where $u_{i}$ is the $i^{\text {th }}$ component of fluid velocity and $\theta$ is the fluid temperature. The constitutive parameters $\alpha_{i}, \beta_{i}$ being polynomials in terms of $d$ and $b$ in which the coefficients depend on fluid density $(\rho)$ and the temperature $(\theta)$. The fluid is called Stokesian fluid if the stress tensor depends on the rate of deformation tensor ' $d$ ' and it is called Fourier-heat-conducting fluid when the heat flux bi-vector depends on the temperature gradient, the coefficients $\alpha_{1}$ and $\alpha_{3}$ may be identified as the fluid pressure 
and coefficient viscosity coefficient respectively and $\alpha_{5}$ as that of cross-viscosity coefficient.

The flow of incompressible thermo-viscous fluids in general satisfies the usual following conservation laws(equations):

Law of conservation of mass(equation of continuity):

$v_{i, i}=0$

Law of conservation of momentum(equation of momentum):

$\rho\left[\frac{\partial v_{i}}{\partial t}+v_{k} v_{i, k}\right]=\rho F_{k}+t_{j i, i}$

and the energy equation(Law of Conservation of energy):

$\rho c \dot{\theta}=t_{i j} d_{i j}-q_{i, i}+\rho \gamma$

where

$F_{k}=k^{\text {th }}$ Component of external force per unit mass,

$c=$ Specific heat,

$\gamma=$ Thermal energy source per unit mass

$q_{i}=i^{\text {th }}$ Component of heat flux bivector $=\in_{i j k} h_{j k} / 2$

$t_{i j}=$ The components of stress tensor

$d_{i j}=$ The components of rate of deformation tensor

The investigation of the flow of thermo-viscous flows has become an important topic due to the recovery of crude oil from the pores of reservoir rocks, the extraction and filtration of oil from wells, the oil reservoir treated by the reservoir engineer, the extraction of energy from geo-thermal regions are some of the areas in which thermo-viscous flows have been noticed. Keeping this in mind the relevance and growing importance of thermo-viscous fluids in geophysical fluid dynamics, chemical technology and industry; the present paper attempts to study the various material parameters effects on Drag, velocity and temperature fields in the steady flow around a moving circular pipe in a thermo-viscous fluid in the absence of pressure gradient.

\section{Mathematical Formulation and Solution}

Let us consider the cylindrical polar coordinate system $(r, \theta, z)$ with the z-axis is along the axis of the pipe and $r$ is the radial distance from the centre of the pipe. The flow is represented by the velocity $(0,0, u(r))$ and the temperature of the fluid $\eta(r)$. This choice 
of velocity satisfies the law of conservation of mass $v_{i, i}=0$. The flow is assumed under the constant temperature gradient and in the absence of pressure gradient.

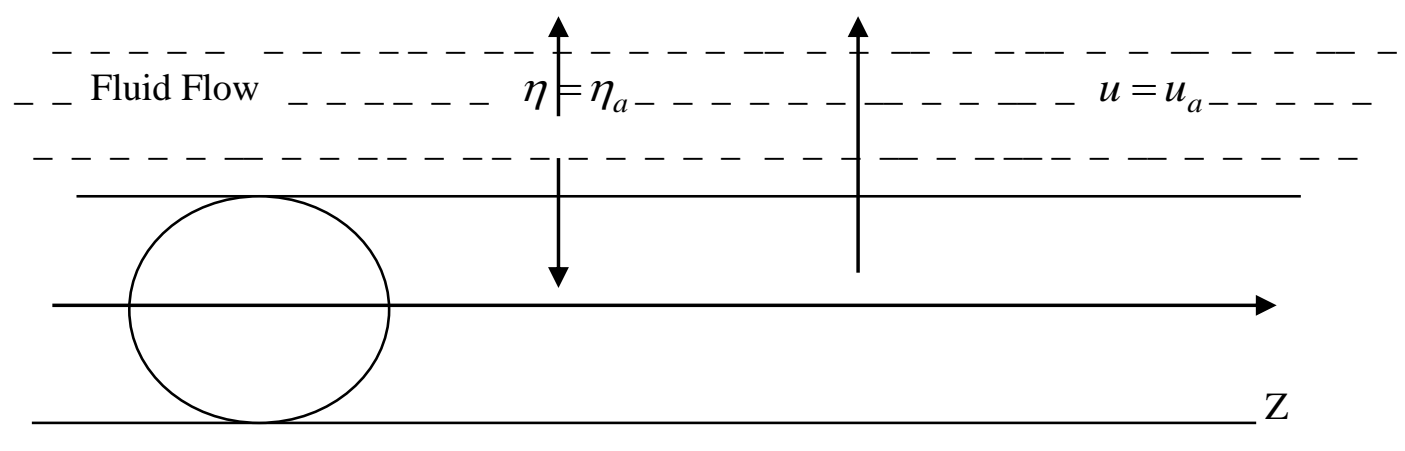

Figure 1. Physical Model

The flow is assumed under the constant temperature gradient and in the absence of pressure gradient. This assumption leads the basic governing equations characterizing the flow are the following:

In the radial direction:

$$
\frac{\partial p}{\partial r}=\mu_{c}\left\{\frac{1}{r}\left(\frac{\partial u}{\partial r}\right)^{2}+2 \frac{\partial u}{\partial r} \frac{\partial^{2} u}{\partial r^{2}}\right\}-\alpha_{6} \frac{1}{r}\left(\frac{\partial \eta}{\partial r}\right)^{2}+\rho F_{r}
$$

In the transverse direction:

$$
\rho F_{\theta}=\alpha_{8}\left\{\frac{\partial u}{\partial r} \nabla^{2} \eta+\frac{\partial \eta}{\partial r} \nabla^{2} u\right\}
$$

In the axial direction(z-direction):

$$
\frac{\partial p}{\partial z}=\mu \nabla^{2} u-\alpha_{6} c_{2} \nabla^{2} \eta+\rho F_{z}
$$

and the energy equation:

$$
\rho c u\left(\frac{\partial \eta}{\partial z}\right)=\mu \frac{1}{r}\left(\frac{\partial u}{\partial r}\right)^{2}-\alpha_{6}\left(\frac{\partial \eta}{\partial z}\right)\left(\frac{\partial u}{\partial r}\right)\left(\frac{\partial \eta}{\partial r}\right)+k \nabla^{2} \eta+\beta_{3}\left(\frac{\partial \eta}{\partial z}\right) \nabla^{2} u+\rho \gamma
$$

The boundary conditions of the problem are:

$$
u(r=a)=u_{a}, u(r=\infty)=\text { finite }
$$

and

$$
\eta(r=a)=\eta_{a}, \eta(r=\infty)=\text { finite }
$$


The flow is generated is assumed in the absence of pressure gradient $\left(\frac{\partial p}{\partial z}\right)$, external force in the Z-direction and the internal energy source. Further, for the slow steady motion of the fluid, the non-linear terms in the equations (3) and (4) could be neglected.

Now the momentum equation in z-direction reduces to

$0=\mu \nabla^{2} u-\alpha_{6} c_{2} \nabla^{2} \eta$

and the energy equation reduces to

$\rho c c_{2} u=k \nabla^{2} \eta+\beta_{3} c_{2} \nabla^{2} u$

The equations (7) and (8) represent the coupled equations in $u$ and $\eta$.

Here $c_{2}\left(=\frac{\partial \eta}{\partial z}\right)$ is the constant temperature gradient, $\alpha_{6}$ is the thermo-stress coefficient and $\beta_{3}$ is called thermal conductivity coefficient.

The boundary conditions of the problem for the flow around the moving circular pipe are

$$
u(r=a)=u_{a}, u(r=\infty)=\text { finite }
$$

and

$$
\eta(r=a)=\eta_{a}, \eta(r=\infty)=\text { finite }
$$

The following non-dimensional quantities are introduced to covert the above equations in non-dimensional form:

$r=a R, u=\frac{\mu}{2 \rho a} U, \quad \eta=\eta_{a} T$

Now the equations of momentum and energy reduces to

$$
\nabla^{2} U=m^{2} U
$$

and

$$
\nabla^{2} T+\frac{\delta_{1} p_{r}}{1+a_{6} b_{3} p_{r}} U=0
$$

Here $m^{2}=\frac{a_{6} p_{r}}{1+a_{6} b_{3} p_{r}}$ with $p_{r}=\frac{\mu c}{k}$ (Prandtl number)

and $\quad \delta_{1}=\frac{c_{2} a}{2 \eta_{\infty}}$ 
The boundary conditions in non-dimensional form are reduced to

$$
U(R=1)=U_{a}, U(R=\infty)=\text { finite }
$$

and

$$
T(R=1)=1, T(R=\infty)=\text { finite }
$$

Solving the equations (11),(12), (13) and (14), we get the velocity and temperature fields as

$$
U(R)=\frac{K_{0}(m R)}{K_{0}(m)} U_{a}
$$

and

$$
T(R)=\frac{\delta_{1} p_{r}}{1+a_{6} b_{3} p_{r}} \frac{1}{m^{2}}\left\{\frac{K_{0}(m R)}{K_{0}(m)}-1\right\} U_{a}+1
$$

Nussult number on the boundary is obtained as:

$$
\left.\frac{\partial T}{\partial R}\right|_{R=1}=\frac{\delta_{1} p_{r}}{1+a_{6} b_{3} p_{r}} \frac{1}{m} \frac{K_{1}(m)}{K_{0}(m)} U_{a}
$$

Drag force on the boundary is obtained as:

$\operatorname{Drag}_{R=1}=2 \pi \frac{K_{1}(m)}{K_{0}(m)}\left\{\frac{-\mu}{2 \rho a} m+\frac{a_{6} c_{2} \eta_{a} \delta_{1} p_{r}}{1+a_{6} b_{3} p_{r}} \frac{1}{m}-1\right\} U_{a}$

Using the equations (15) and (16) in the equation (1) in the absence of external force in the radial direction. The Pressure Distribution is obtained as:

$$
P=\frac{1}{K_{0}^{2}(m)}\left\{\begin{array}{l}
\frac{\mu_{c} \mu^{2}}{8 \rho^{2} a^{4}} m^{2}\left[2 K_{0}{ }^{2}(m R)-3 K_{1}^{2}(m R)\right]- \\
\frac{\alpha_{6} \eta_{a}^{2}}{2 m^{2} a^{2}}\left(\frac{\delta_{1} p_{r}}{1+a_{6} b_{3} p_{r}}\right)^{2}\left[K_{0}{ }^{2}(m R)-K_{1}{ }^{2}(m R)\right]-1
\end{array}\right\} W_{a}^{2}+P_{0}
$$

It is observed that the pressure distribution is effected by the physical parameters such as the coefficient of (Reiner-Rivlin) cross-viscosity $\left(\mu_{c}\right)$,Thermo mechanical stress interaction coefficient $\left(a_{6}\right)$, Strain thermal conductivity coefficient $\left(b_{3}\right)$ and the prandtl number $\left(p_{r}\right)$. 


\section{Graphs}

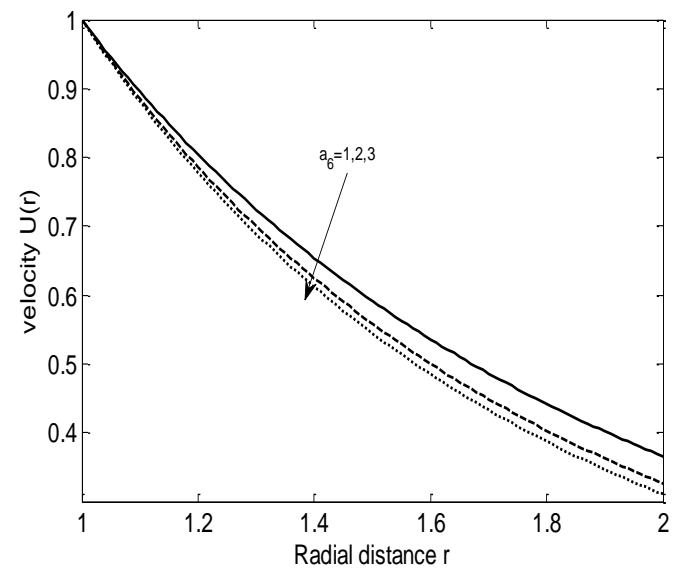

Figure 2. Velocity versus $a_{6}$ and

$$
b_{3}=1
$$

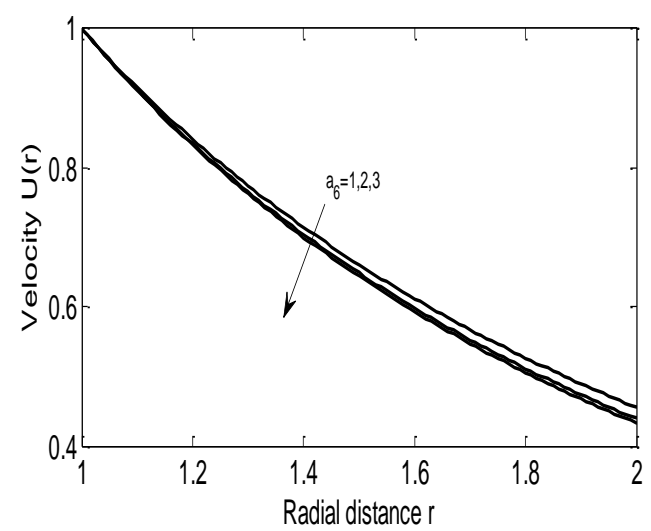

Figure 4. Velocity versus $a_{6}$ and

$$
b_{3}=5
$$

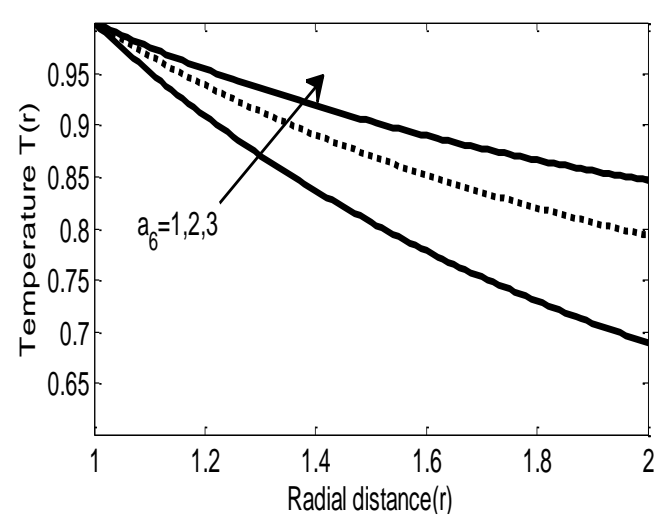

Figure 6. Temperature versus $a_{6}$ and

$$
b_{3}=3
$$

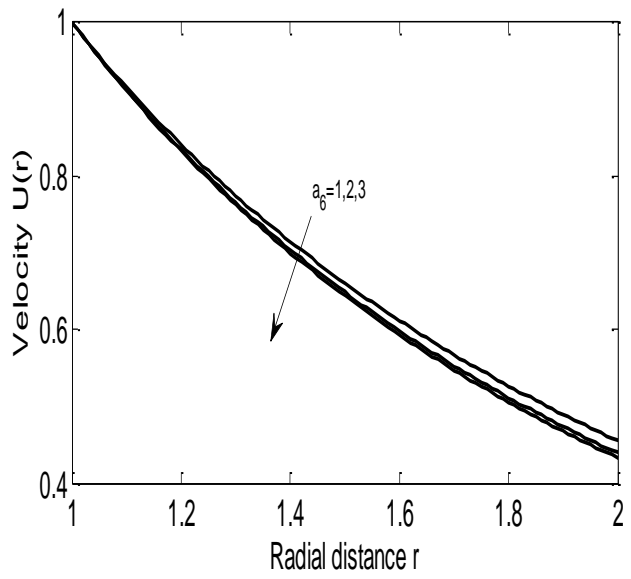

Figure 3. Velocity versus $a_{6}$ and $b_{3}=3$

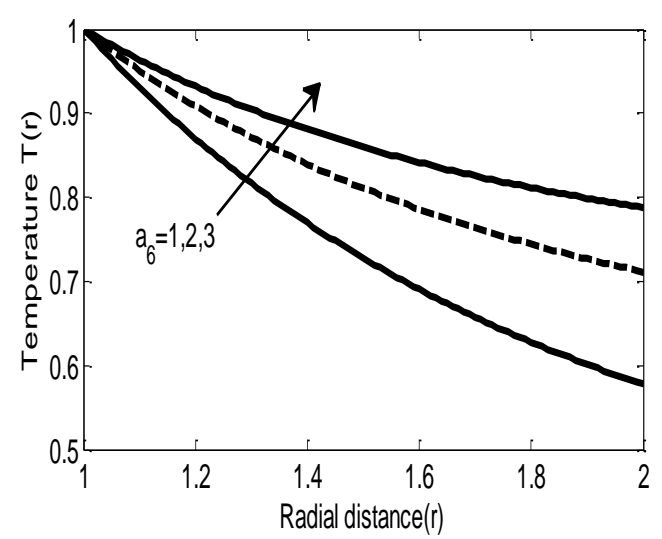

Figure 5. Temperature versus $a_{6}$ and

$$
b_{3}=1
$$

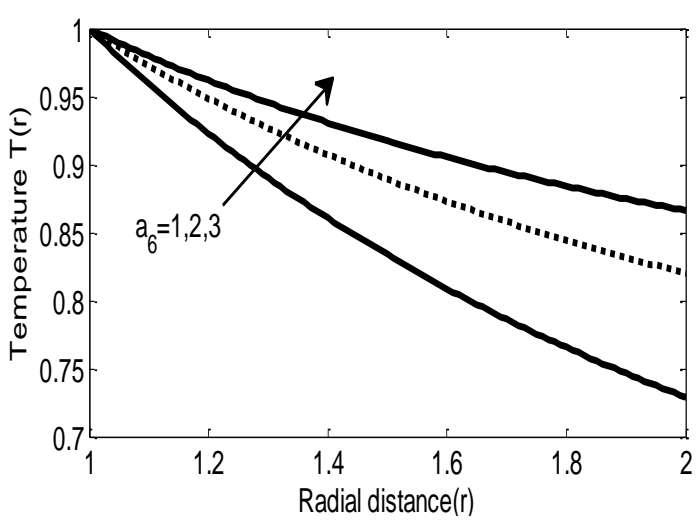

Figure 7.Temperature versus $a_{6}$ and

$$
b_{3}=5
$$




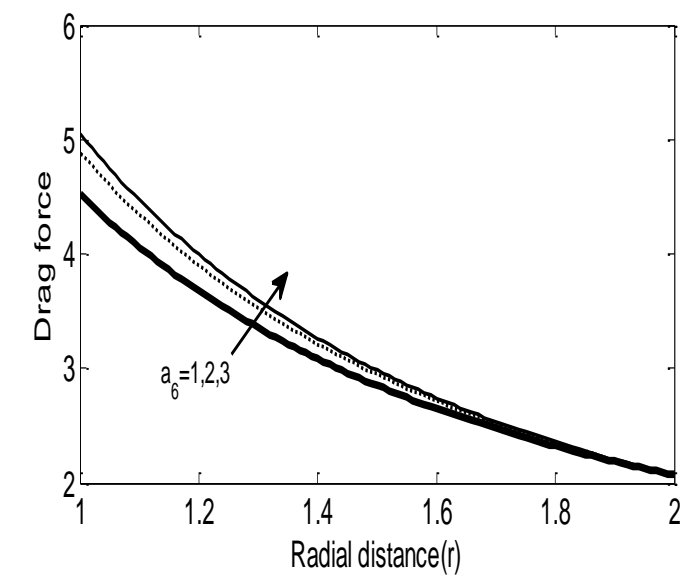

Figure 8. Drag versus $a_{6}$ and

$$
b_{3}=1
$$

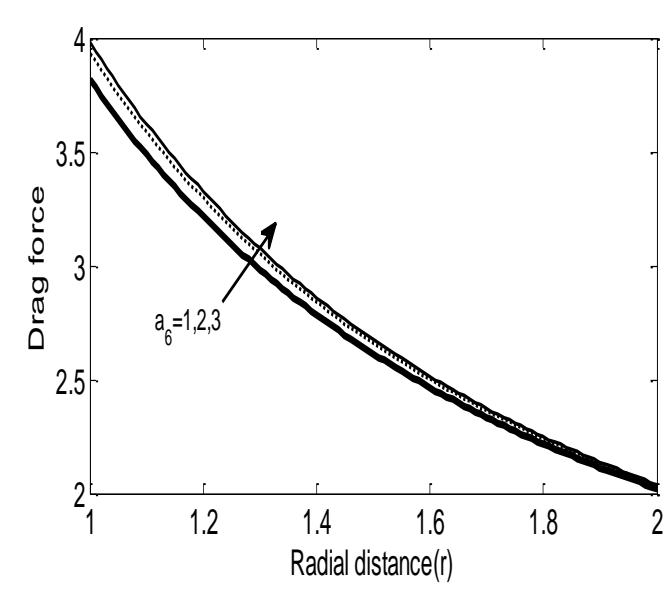

Figure 9. Drag versus $a_{6}$ and

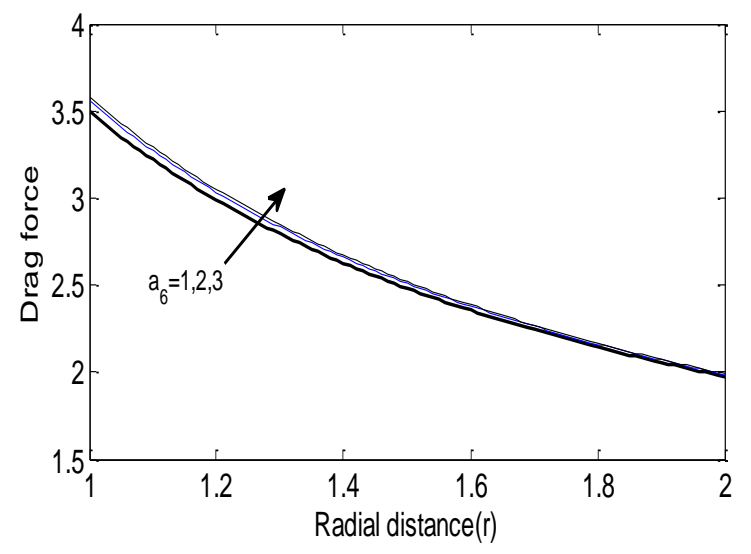

Figure 10. Drag versus $a_{6}$ and $b_{3}=5$

\section{Results and Discussion}

The flow under the assumption of slow steady motion of a fluid is considered and this paper deals with steady flow around a moving circular pipe in a Thermo-viscous fluid in the absence of pressure gradient with the constant temperature gradient.

The effects of material parameters such as Strain thermal conductivity coefficient $\left(b_{3}\right)$, and thermo-mechanical stress interaction coefficient on the velocity field, temperature distribution and on the Drag have been illustrated for the fixed values $\mu=1, C_{2}=1$, $p_{r}=1, \delta_{1}=1$ and $a_{1}=1$.

The variations of velocity profiles for different values of Strain thermal conductivity coefficient $\left(b_{3}\right)$, and thermo-mechanical stress interaction coefficient $\left(a_{6}\right)$ are shown in Figures (2-4). The variations of temperature profiles for different values of Strain thermal conductivity coefficient $\left(b_{3}\right)$, and thermo-mechanical stress interaction coefficient $\left(a_{6}\right)$ are shown in Figures (5-7). The Drag variations of the fluid for different values of Strain thermal conductivity coefficient $\left(b_{3}\right)$, and thermo-mechanical stress interaction coefficient $\left(a_{6}\right)$ are shown in Figures (8-10).

From the Figures (2-4), it is observed that the velocity of the fluid decreases at the slower rate as the value of thermo-mechanical stress interaction coefficient $\left(a_{6}\right)$ 
increases. The Figures (2-4) also depicts that, the velocity of the fluid increases as the values of Strain thermal conductivity coefficient $\left(b_{3}\right)$ increases.

It is noticed from the Figures (5-7) that the temperature of the fluid increases at the faster rate as the value of thermo-mechanical stress interaction coefficient $\left(a_{6}\right)$ and Strain thermal conductivity coefficient $\left(b_{3}\right)$ increases.

From the Figures (8-10), it is observed that the Drag of the fluid increases at the slower rate as the value of thermo-mechanical stress interaction coefficient $\left(a_{6}\right)$ increases. From the Figures (8-10) it is observed that, the Drag of the fluid decreases as the values of Strain thermal conductivity coefficient $\left(b_{3}\right)$ increases.

From the Figures (2-10), it is found that the velocity of the fluid, for the various physical parameters of the flow, the temperature of the fluid and the Drag of the fluid decreases as the flow is far away from the circular pipe and this presents the physical insight of the problem.

\section{Conclusions}

The steady flow of a thermo-viscous fluid around a moving circular pipe in the absence of pressure gradient with the constant temperature gradient is considered. The following conclusions are drawn from the above illustrations:

1. Increasing the value of thermo-stress viscosity coefficient decreases the velocity of the fluid and increasing the value of thermo conductivity coefficient increases the velocity of the fluid around the moving circular plate.

2. The temperature of the fluid increases at faster rate for increasing both the values of thermo-stress viscosity coefficient and thermo conductivity coefficient.

3. The Drag on the boundary of moving circular plate increases for increasing the value of thermo-stress viscosity coefficient and decreases for increasing the thermo conductivity coefficient.

\section{References}

[1] K. Anuradha, "On steady and unsteady flows of thermo-viscous Fluids", Ph. D thesis, J.N.T.U. Hyderabad, (2006).

[2] A. E. Green and P. M. Naghdi, "A dynamical theory of interacting Continua”, International Journal of Engg. Sci., vol. 3, (1965), pp. 231-241.

[3] A. E. Green and P. M. Naghdi, "A new thermo-viscous theory for fluids", Journal of Non-Newtonian Fluid Mechanics, vol. 56, no. 3, (1995) March, pp. 289-306.

[4] P. D. Kelly, "Some viscometric flows of incompressible thermo-viscous fluids", International Journal of Engg. Sci., vol. 2, (1965), pp. 519-537.

[5] S. L. Koh and A. C. Eringin, "On the foundations of non-linear thermo-elastic fluids", International Journal of Engg. Sci., vol. 1, (1963), pp. 199-229.

[6] R. Muthuraj and S. Srinivas, "Flow of a Thermoviscous Fluid through an Annular Tube with Constriction”, Defence Science Journal, vol. 57, no. 5, (2007) September, pp. 653-659.

[7] E. Nagaratnam, "Some steady and unsteady flows of thermo- viscous fluids", Ph. D thesis, J.N.T.U Hyderabad, (2006).

[8] P. Nageswara Rao, "Some problems in thermo-viscous fluid Dynamics", Ph. D thesis, K.U. Warangal, (1979).

[9] P. Nageswara Rao and N.Ch. Pattabhi Ramacharyulu, "Steady flow of a second order thermo- viscous fluid over an infinite plate", Proc. Ind. Acad. Sci., vol. 88A, Part III No. 2, (1979), pp .157-162.

[10] P. Nageswara Rao and N.Ch. Pattabhi Ramacharyulu, "Steady flow of a thermo-viscous fluid through straight tubes”, Journal of Ind. Inst. Sci., vol. 61(B), (1979) June, pp. 89-102.

[11] P. Nageswara Rao and N.Ch. Pattabhi Ramacharyulu, "A note on steady slow motion of thermo-viscous fluid through a circular tube", Indian journal of pure and appl. Math., vol. 11, no. 4, (1980) April, pp. 487-491.

[12] N. Ch. Pattabhi Ramacharyulu, "Problems in the steady of non-Newtonian Fluid Dynamics", Ph. D thesis, O. U. Hyderabad, (1966). 
[13] N. Pothanna, P. Nageswara rao and N.Ch. Pattabhi Ramacharyulu, "Flow of slightly thermo-viscous fluid in a porous slab bounded between two permeable parallel plates", Int. J. Adv. Appl. Math. and Mech., vol. 2, no. 3, (2015), pp. 1-9.

[14] N. Pothanna, J. Srinivas, P. Nageswara rao and N.Ch. Pattabhi Ramacharyulu, "Linearization of thermoviscous fluid in a porous slab bounded between two fixed permeable horizontal parallel plates in the absence of thermo-mechanical interaction coefficient", International Journal of Modern Trends in Engineering and Research, vol. 1, no. 5, (2014), pp. 412-424.

[15] N. Pothanna, J. Srinivas and P. Aparna, "Unsteady Forced Oscillations of a fluid bounded by rigid bottom", International Journal of Control Theory and Applications, vol. 9, no. 19, (2016), pp. 90499054.

[16] J. Srinivas, N. Pothanna, P. Nageswara rao and N.Ch. Pattabhi Ramacharyulu, "Slow steady motion of a thermo-viscous fluid between two parallel plates with constant pressure and temperature gradients", International Journal of Research in Engineering and Technology, vol. 2, no. 11, (2013), pp. 294-299. 